\title{
Antifungal susceptibility testing of vulvovaginal Candida species among women attending antenatal clinic in tertiary care hospitals of Peshawar
}

This article was published in the following Dove Press journal: Infection and Drug Resistance

\author{
Maria Khan' \\ Jawad Ahmed ${ }^{2}$ \\ Amina $\mathrm{Gul}^{3}$ \\ Aamer Ikram' \\ Farida Khurram Lalani' \\ 'Department of Microbiology, Armed \\ Forces Institute of Pathology, National \\ University of Management Sciences, \\ CMH Rawalpindi, Rawalpindi, Pakistan; \\ ${ }^{2}$ Department of Microbiology, \\ Institute of Basic Medical Sciences, \\ Khyber Medical University, \\ Peshawar, Pakistan; ${ }^{3}$ Department of \\ Microbiology, Khyber Medical College, \\ Khyber Medical University, Peshawar, \\ Pakistan
}

Background: Vulvovaginal candidiasis (VVC) is considered as a pervasive gynecological problem among women worldwide. Owing to this fact, in the current study, we aimed at assessing the prevalence rate of Candida spp. causing VVC in symptomatic pregnant women and their antifungal susceptibility pattern.

Methods: This study was carried out in the tertiary care hospitals of Peshawar during the period of July 1, 2016 to December 31, 2016. The study group included 450 pregnant women in the age group of 17-44 years with symptoms of excessive vaginal discharge, pain and pruritis. In all, 108 pregnant women were culture positive for Candida. Antimicrobial susceptibility testing (AST) was conducted on specimens against various azoles and polyene $\mathrm{F}$ group of antifungals. Results: Out of 108 Candida spp. isolated from vaginal swabs, there were 45 (41.7\%) Candida albicans, 18 (16.7\%) Candida tropicalis, 18 (16.7\%) Candida krusei, 16 (14.8\%) Candida glabrata and 11 (10.2\%) Candida dubliniensis. According to age distribution, 27 years was the mean age. Pregnancy trimester distribution among patients was as follows: 21 (19.4\%) patients were in their first trimester, $65(60.2 \%)$ patients were in their second trimester and $22(20.4 \%)$ patients were in the third trimester. Susceptibility of fluconazole was determined as follows: $33.3 \%$ of the Candida isolates were sensitive, $4.6 \%$ were susceptible dose dependent (SDD) and $62 \%$ were resistant. Susceptibility of Candida spp. with respect to nystatin in patients with VVC was as follows: $25 \%$ were sensitive, $16.7 \%$ were SDD and $58.3 \%$ were resistant. Susceptibility of clotrimazole was analyzed, and it was sensitive in $21.3 \%$ of patients, SDD in $19.4 \%$ of patients and resistant in $59.3 \%$ of patients. Voriconazole susceptibility was recorded to be sensitive in $85.2 \%$ of patients, SDD in $4.6 \%$ of patients and resistant in $10.2 \%$ of patients suffering from VVC. Susceptibility results for itraconazole in patients with VVC were as follows: $42.6 \%$ of patients were sensitive, $16.7 \%$ of patients were SDD, and $40.7 \%$ of patients were resistant.

Conclusion: In this study, frequency of VVC was noted to be high in the second trimester of pregnancy, with the highest frequency of $C$. albicans isolated, followed by $C$. tropicalis and C. krusei. Antifungal susceptibility testing revealed that fluconazole was exceedingly resistant against Candida species (62\%), followed by clotrimazole (59.3\%) and nystatin (58.3\%). On the contrary, voriconazole had the highest antimicrobial activity against Candida species $(85.2 \%)$. Keywords: vulvovaginal candidiasis, fluconazole, voriconazole, itraconazole, ketoconazole, nystatin

\section{Plain language summary}

Problem: Resistance to antifungal agents has represented a major challenge for the clinic and a major public health problem, especially if the patient has been treated previously with antifungal agents.
Department of Microbiology, Armed Forces Institute of Pathology, National University of Management Sciences, $\mathrm{CMH}$ Rawalpindi, Rawalpindi, Pakistan Tel +9233 191। 1986 Email kmaria22@hotmail.com 
What is already known? Vulvovaginal candidiasis (VVC) is the most frequent gynecological ruling among women of childbearing age. Among sexually active women, $75 \%$ have at least once experienced symptomatic VVC, which usually presents as soreness, burning, itching and abnormal curd-like vaginal discharge.

What this paper adds? With multiple antifungals and varying susceptibility patterns of Candida, the study was conducted to determine the frequency of common Candida species among women with VVC and also the antifungal susceptibility pattern of identified Candida species causing VVC and make information accessible to the clinicians for effectual therapeutic outcome.

\section{Introduction}

Globally amid the fungal infections in human beings, candidal infections are predominantly reported. More than 200 species of Candida have been reported. ${ }^{1}$ In healthy human beings, Candida is considered as a commensal, and its scope to produce either superficial or systemic infections depends on the host immune system and various risk factors. ${ }^{2}$ Vulvovaginal candidiasis (VVC) is the most frequent gynecological ruling among women of childbearing age. ${ }^{3}$ Among sexually active women, $75 \%$ have at least once experienced symptomatic VVC, which usually presents as soreness, burning, itching and abnormal curd-like vaginal discharge. ${ }^{4,5}$ Pregnancy was the commonest factor (55\%) followed by usage of broad-spectrum antibiotics $(8 \%)$. Other risk factors were use of oral contraceptive pills, diabetes mellitus and tuberculosis. ${ }^{6}$ In pregnant women, VVC complications include abortion, chorioamnionitis and subsequent preterm delivery. $^{7}$ Transmission of Candida can take place from the infected mother's vagina to the newborn, leading to Candida congenital infection. ${ }^{8}$ It is perceived that Candida albicans presented for VVC cases of 70-90\%, with a recent surfacing of non-albicans species. $^{9}$

The improvement of standardized antimicrobial susceptibility testing (AST) has been the area under discussion of several studies in the past few decades. Reference scheme for yeast susceptibility testing (National Committee for Clinical Laboratory Standards [NCCLS]) is available. Agar-based susceptibility testing procedure has been a focus of interest for many researchers and comprises the classical disk diffusion method along with novel E-test method. ${ }^{10,11}$ Antifungal susceptibility testing may specify clinical response, predict treatment failure and develop local antibiograms, aiding in empirical selection of antifungals. ${ }^{12}$ Resistance to antifungal agents has represented a major challenge in public health problems, particularly if the patient has been in the past treated with an azole group of antifungal agent; the likelihood of microbiological resistance should likely be considered. With a diverse range of antifungals use and anecdotal susceptibility patterns of Candida, it has at the present turn out to be indispensable to perform AST and make information accessible to the clinicians for effectual therapeutic outcome.

\section{Methods}

The study was conducted at antenatal units of tertiary care hospitals in Peshawar. Samples were processed at the Department of Microbiology, Institute of Basic Medical Sciences (IBMS), Khyber Medical University, Peshawar, from July 2016 to December 2016. Permission from the institutional ethical committee of Khyber Medical University was obtained before commencement of the study. An informed consent form was obtained from the participants after the contents of the form were clearly explained. Furthermore, they were assessed on the basis of age, pregnancy trimester and signs/symptoms during the gynecological examination. The participants younger than 18 years were able to sign informed consent on their own behalf, and this was acceptable to the institutional ethical committee of Khyber Medical University. Both symptomatic and non-symptomatic pregnant women's samples that were colonized or presenting with VVC, i.e., pruritus, leukorrhea, edema and vulvovaginal erythema presenting with whitish plaques on the mucosa were used for mycological cultivation. Samples were collected upon inserting a sterile vaginal speculum into the vagina; two high vaginal swabs, one after the other, were taken by a sterile cotton wool swab into the posterior vaginal fornix and rotated gently. The swabs were then inserted into its outer casing and were labeled with the patient's case number, name and date. One of the swabs was used for direct smear examination, and the second one was inoculated on Sabouraud dextrose agar (SDA) and incubated aerobically at $37^{\circ} \mathrm{C}$.

Examination of high vaginal swabs was done by the wet preparation method, i.e., $10 \%$ potassium hydroxide $(\mathrm{KOH})$ preparation and Gram staining. Sterile normal saline drops were added to one of the tubes and shaken to extricate materials from the swab. Then, a wet film was prepared by putting a drop of the saline deposit onto a clean glass slide, which was then covered with a cover slip and examined under the microscope for budding yeast cells and pseudohyphae using $40 \times$ objective lens. Thereafter, a sterile inoculating loop was used to transfer small amount of the deposit onto a clean glass slide to form a smear. The smear was air-dried, and then, Gram staining was performed. The slide was then examined under the microscope using the oil immersion objective lens for yeast cells. 
For phenotypic identification, the second swab was streaked onto culture plates of SDA (Oxoid, Basingstoke, UK) and Sabouraud dextrose agar with chloramphenicol (SC; Oxoid), which was then incubated for 48 hours at $27^{\circ} \mathrm{C}$. The isolated pure colonies were confirmed on Gram staining for yeast cells.

Candida spp. was isolated on CHROMagar Candida (Oxoid) which is a differential and selective medium. Isolated Candida colonies on SDA were subcultured onto CHROMagar using an inoculating loop and incubated at $37^{\circ} \mathrm{C}$ for 24 hours. Presumptive identification was done based on colony color of the growing Candida strains. Identification of Candida was based on the color of individual colony. API 20C AUX (bioMérieux, Marcy-l'Étoile, France) was further used for species identification and confirmation, which is based on 19 carbohydrate assimilation tests plus a negative control, read by assessing cupules for turbidity. The kit was used in accordance with the guidelines given by the manufacturer. Reading of the strips was done after 48 and 72 hours of incubation at $30^{\circ} \mathrm{C}$. Using this method, the following Candida species were identified: C. albicans (green colonies), Candida krusei (pink colonies) Candida glabrata (purple colonies), Candida tropicalis (blue colonies) and C. dublinensis (darker green colonies).

\section{Antifungal susceptibility testing}

A suspension was prepared by picking five to six colonies from the SDA culture plate of $\sim 1 \mathrm{~mm}$ diameter from a 24-hour old culture of Candida species. Colonies were then inoculated in $5 \mathrm{~mL}$ of sterile saline, and its turbidity was adjusted to $0.5 \mathrm{McFarland}$ standards visually. A sterile cotton wool swab was moistened in the adjusted inoculum suspension, and then, excess fluid was rinsed by rolling the swab on the inside surface of the tube above the fluid surface. Müller-Hinton agar (MHA) surface was streaked to make a lawn of the isolate.

Antifungal susceptibility testing was undertaken by the disk diffusion method. Using disk dispenser (Oxoid ${ }^{\mathrm{TM}}$ ), fluconazole disk $(10 \mu \mathrm{g})$, itraconazole $(10 \mu \mathrm{g})$, voriconazole $(10 \mu \mathrm{g})$, clotrimazole $(10 \mu \mathrm{g})$ and nystatin (100 IU) antifungal discs (Thermo Scientific ${ }^{\mathrm{TM}}$ Oxoid $^{\mathrm{TM}}$ ) were applied on MHA (Thermo Scientific ${ }^{\mathrm{TM}}$ Oxoid $^{\mathrm{TM}}$ ) as recommended by the Clinical Laboratory Standard Institute (CLSI) M44A document.

The plates were incubated in ambient air at $35^{\circ} \mathrm{C}$ and read at 24 hours. The diameters of zones of inhibition were measured in millimeters using a ruler for each antifungal disk. Interpretation of all antifungal susceptibility (susceptible $\mathrm{S}$,
Table I Interpretative breakpoints of antifungal agents

\begin{tabular}{lllll}
\hline Antifungals & $\begin{array}{l}\text { Disk } \\
\text { concentration }\end{array}$ & \multicolumn{3}{l}{ Zone of activity $(\mathbf{m m})$} \\
\cline { 3 - 5 } & & \multicolumn{4}{c}{$\begin{array}{l}\text { Sensitive } \\
\text { Intermediatel } \\
\text { SDD }\end{array}$} \\
\hline Nystatin & $100 \mathrm{U}$ & $\geq 15$ & $10-14$ & $\leq 10$ \\
Clotrimazole & $10 \mu \mathrm{g}$ & $\geq 20$ & $12-19$ & $\leq 11$ \\
Fluconazole & $10 \mu \mathrm{g}$ & $\geq 19$ & $15-18$ & $\leq 14$ \\
Voriconazole & $10 \mu \mathrm{g}$ & $\geq 19$ & $15-18$ & $\leq 14$ \\
Itraconazole & $10 \mu \mathrm{g}$ & $\geq 15$ & $10-14$ & $\leq 9$ \\
\hline
\end{tabular}

Abbreviation: SDD, susceptible dose dependent.

susceptible dose dependent [SDD], and resistant R) was done according to CLSI standards (Table 1). Quality control was undertaken by using quality control strains, American Type Culture Collection (ATCC) 90028.

\section{Results}

A total of 108 patients were observed to determine frequency of in vitro susceptibility of Candida spp., and the results were analyzed.

Age distribution among 108 patients was analyzed as follows: $11(10.1 \%)$ patients were in age range $17-20$ years, $28(25.9 \%)$ patients were in age range $21-24$ years, 31 (28.7\%) patients were in age range in $25-28$ years, 20 $(18.52 \%)$ patients were in age range $29-32$ years, 12 $(11.11 \%)$ patients were in age range 33-36 years, three $(2.7 \%)$ patients were in age range $37-40$ years and three (2.7\%) patients were in age range $41-44$ years. The minimum age calculated was 17 years, and the maximum age was 44 years. Mean age was 27 years with standard deviation (SD) \pm 5.61012 (Figure 1).

Pregnancy trimester distribution among patients was analyzed as follows: $21(19.4 \%)$ patients were in their first trimester, $65(60.2 \%)$ patients were in their second trimester and $22(20.4 \%)$ patients were in their third trimester (Figure 2).

Frequency of Candida spp. distribution among patients with VVC was analyzed as follows: 45 (41.7\%) patients had isolated C. albicans, 18 (16.7\%) patients had C. tropicalis, 18 (16.7\%) patients had C. krusei, 16 (14.8\%) patients had C. glabrata and 11 (10.2\%) patients had isolated C. dubliniensis. In all, $65 \%$ of the patients had a past history of antifungal use.

Susceptibility of Candida spp. with respect to fluconazole was analyzed as follows: fluconazole was sensitive in $36(33.3 \%)$ of patients, SDD in five (4.6\%) of patients and resistant in 67 (62\%) of patients with VVC (Table 2). Mean zone of inhibition for fluconazole disk was $13 \mathrm{~mm}$ with SD \pm 6.65060 . 


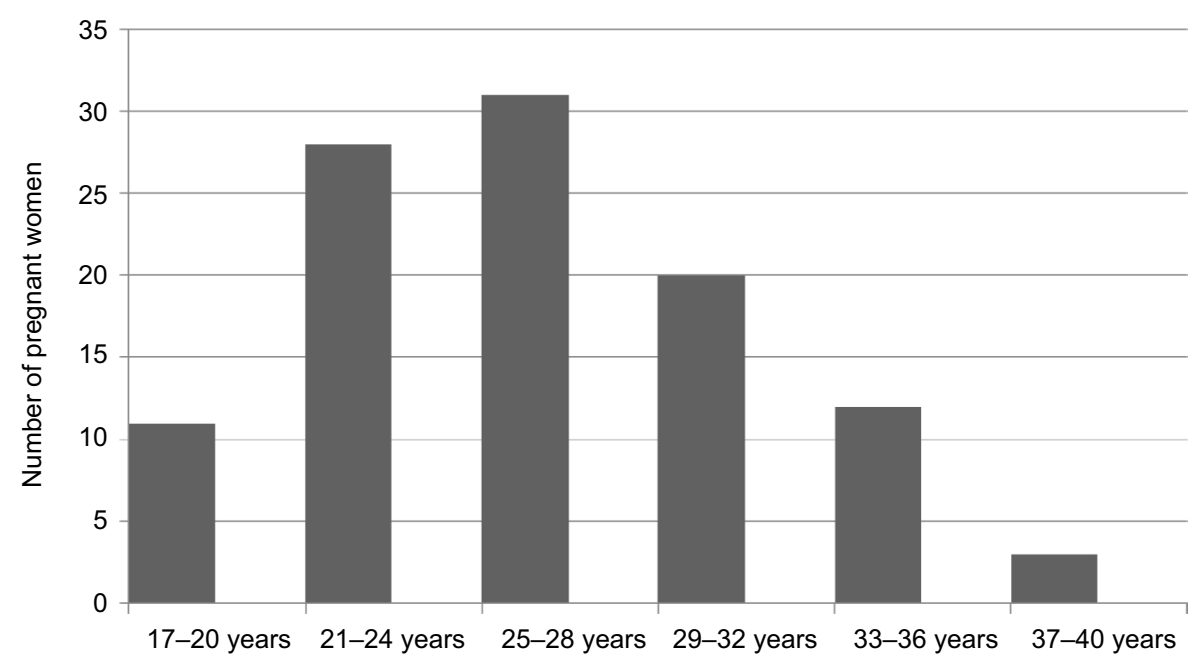

Figure I Age distribution of patients.

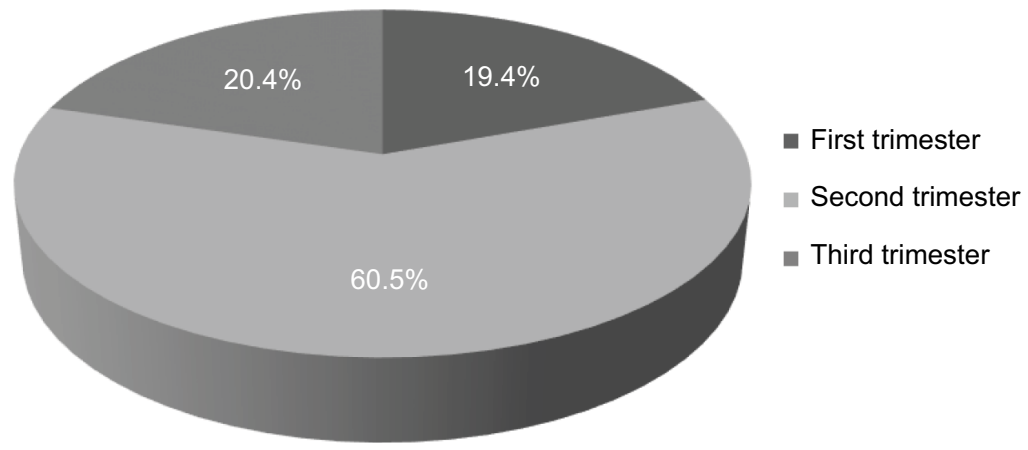

Figure 2 Pregnancy trimester distribution for vulvovaginal candidiasis.

Table 2 Antifungal susceptibility pattern of isolated Candida spp.

\begin{tabular}{llll}
\hline Drugs & Sensitive (\%) & SDD (\%) & Resistant (\%) \\
\hline Fluconazole & 33.3 & 4.6 & 62 \\
Nystatin & 25 & 16.7 & 58.3 \\
Clotrimazole & 21.3 & 19.4 & 59.3 \\
Voriconazole & 85.2 & 4.6 & 10.2 \\
Itraconazole & 42.6 & 16.7 & 40.7 \\
\hline
\end{tabular}

Abbreviation: SDD, susceptible dose dependent.

Susceptibility of Candida spp. with respect to nystatin was analyzed as follows: nystatin was sensitive in $27(25 \%)$ of Candida isolates, SDD in 18 (16.7\%) of Candida isolates and resistant in 63 (58.3\%) of Candida isolates causing VVC (Table 2). Mean zone of inhibition for nystatin disk was $10 \mathrm{~mm}$ with $\mathrm{SD} \pm 4.58981$.

Susceptibility of Candida spp. with respect to clotrimazole was analyzed as follows: clotrimazole was sensitive in 23 (21.3\%) of Candida isolates, SDD in 21 (19.4\%) of Candida isolates and resistant in 64 (59.3\%) of Candida isolates in patients with VVC (Table 2). Mean zone of inhibition for clotrimazole disk was $12 \mathrm{~mm}$ with $\mathrm{SD} \pm 6.56882$.

Susceptibility of Candida spp. with respect to voriconazole was analyzed as follows: voriconazole was sensitive in 92 (85.2\%) of Candida isolates, SDD in five (4.6\%) of Candida isolates and resistant in 11 (10.2\%) of Candida isolates (Table 2). Mean zone of inhibition for voriconazole disk was $21 \mathrm{~mm}$ with $\mathrm{SD} \pm 4.23074$.

Susceptibility of Candida spp. with respect to itraconazole was analyzed as follows: itraconazole was sensitive in $46(42.6 \%)$ of Candida isolates, SDD in $18(16.7 \%)$ of Candida isolates and resistant in 44 (40.7\%) of Candida isolates (Table 2). Mean zone of inhibition for itraconazole disk was $13 \mathrm{~mm}$ with $\mathrm{SD} \pm 5.51711$.

Susceptibility of 45 C. albicans isolates with respect to each of the antifungal was analyzed as follows: fluconazole was sensitive in 17 (37.7\%) of Candida isolates, intermediate 
in four (8.8\%) of Candida isolates and resistant in $24(53.3 \%)$ of C. albicans isolates. Nystatin was sensitive in $12(26.6 \%)$ of $C$. albicans isolates, intermediate in eight (17.7\%) of $C$. albicans isolates and resistant in 25 (55.5\%) of C. albicans isolates. Clotrimazole was sensitive in eight $(17.7 \%)$ of the isolated C. albicans, SDD in 10 (22.2\%) of the isolated $C$. albicans and resistant in $27(60 \%)$ of the isolated C. albicans. Voriconazole was sensitive in $39(86.6 \%)$ of the isolated $C$. albicans, SDD in three (6.6\%) of the isolated C. albicans and resistant in three (6.6\%) of the isolated C. albicans. Itraconazole was sensitive in $24(53.3 \%)$ of the isolated C. albicans, SDD in eight (17.7\%) of the isolated C. albicans and resistant in 13 (28.8\%) of the isolated C. albicans (Figure 3).

Susceptibility of 18 C. tropicalis isolates with respect to each of the antifungal was analyzed as follows: fluconazole was sensitive in 11 (61.1\%) of Candida isolates, SDD in none of the isolated C. tropicalis and resistant in seven (38.8\%) of the isolated C. tropicalis. Nystatin was sensitive in seven (38.8\%) of the isolated C. tropicalis, SDD in four (22.2\%) of the isolated C. tropicalis and resistant in seven (38.8\%) of the isolated $C$. tropicalis. Clotrimazole was sensitive in five (27.7\%) of the isolated C. tropicalis, SDD in six (33.3\%) of the isolated C. tropicalis and resistant in seven (38.8\%) of the isolated $C$. tropicalis. Voriconazole was sensitive in 15 (83.3\%) of the isolated C. tropicalis, SDD in two (11.1\%) of the isolated C. tropicalis and resistant in one (5.5\%) of the isolated C. tropicalis. Itraconazole was sensitive in 11 (61.1\%) of the isolated C. tropicalis, SDD in three (16.6\%) of the isolated C. tropicalis and resistant in four (22.2\%) of the isolated C. tropicalis (Figure 4).
Susceptibility of 18 C. krusei isolated with respect to each of the antifungal was analyzed as follows: fluconazole was sensitive in none of the patients, SDD in none of the patients and resistant in $18(100 \%)$ of the patients isolated with C. krusei. Nystatin was sensitive in five (27.7\%) of the isolated C. krusei, SDD in four (22.2\%) of the isolated $C$. krusei and resistant in nine (50\%) of the isolated C. krusei. Clotrimazole was sensitive in five $(27.7 \%)$ of the isolated $C$. krusei, SDD in three (16.7\%) of the isolated C. krusei and resistant in $10(55.5 \%)$ of the isolated $C$. krusei. Voriconazole was sensitive in $16(88.8 \%)$ of the isolated $C$. krusei, SDD in none of the isolated C. krusei and resistant in two (11.1\%) of the isolated C. krusei. Itraconazole was sensitive in five (27.7\%) of the isolated C. krusei, SDD in one (5.5\%) of the isolated C. krusei and resistant in 12 (66.6\%) of the isolated C. krusei (Figure 5).

Susceptibility of 16 isolates of C. glabrata with respect to each of the antifungal was analyzed as follows: fluconazole was sensitive in six (37.5\%) of the Candida isolates, SDD in none of the Candida isolates and resistant in 10 (62.5\%) of the Candida isolates isolated with C. glabrata. Nystatin was sensitive in three (18.7\%) of the isolated C. glabrata, SDD in two (12.5\%) of the isolated C. glabrata and resistant in $11(68.7 \%)$ of the isolated C. glabrata. Clotrimazole was sensitive in five (31.25\%) of the isolated C. glabrata, SDD in one $(6.25 \%)$ of the isolated C. glabrata and resistant in 10 $(62.5 \%)$ of the isolated C. glabrata. Voriconazole was sensitive in $13(81.25 \%)$ of the isolated C. glabrata, SDD in none of the isolated C. glabrata and resistant in three (18.7\%) of the isolated C. glabrata. Itraconazole was sensitive in four

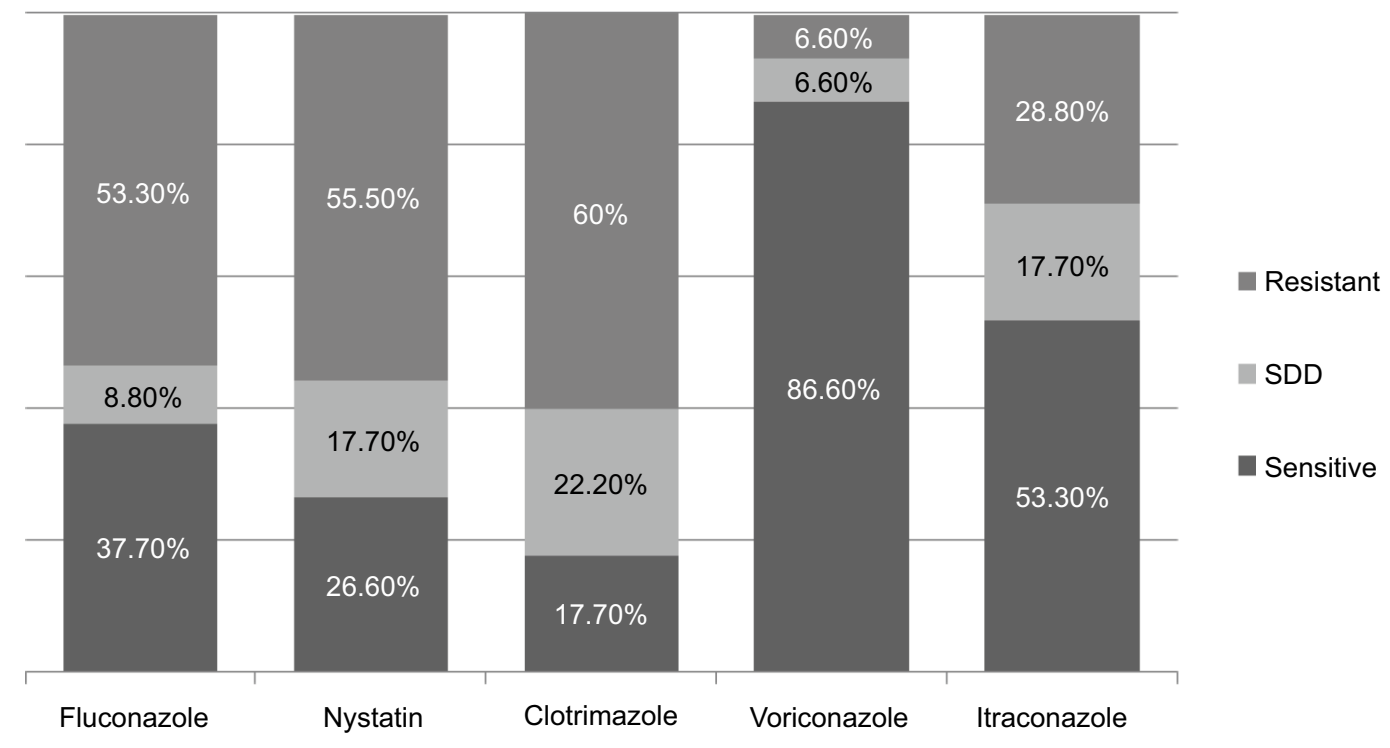

Figure 3 Antifungal susceptibility pattern in Candida albicans. Abbreviation: SDD, susceptible dose dependent. 


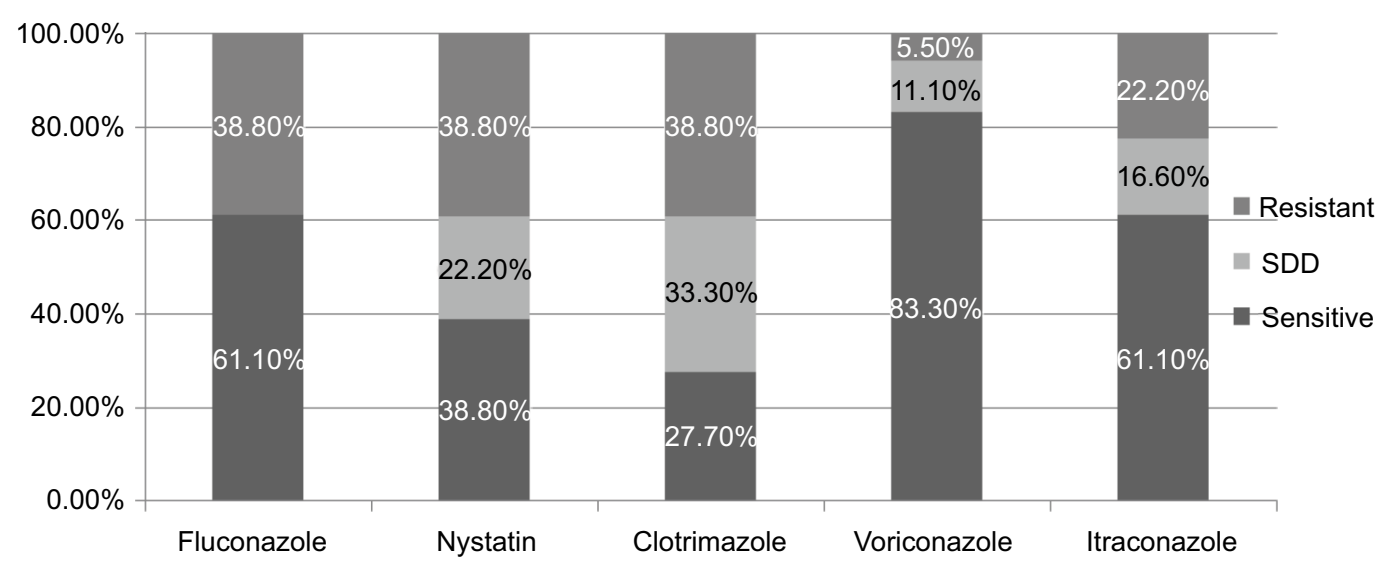

Figure 4 Antifungal susceptibility pattern in Candida tropicalis.

Abbreviation: SDD, susceptible dose dependent.

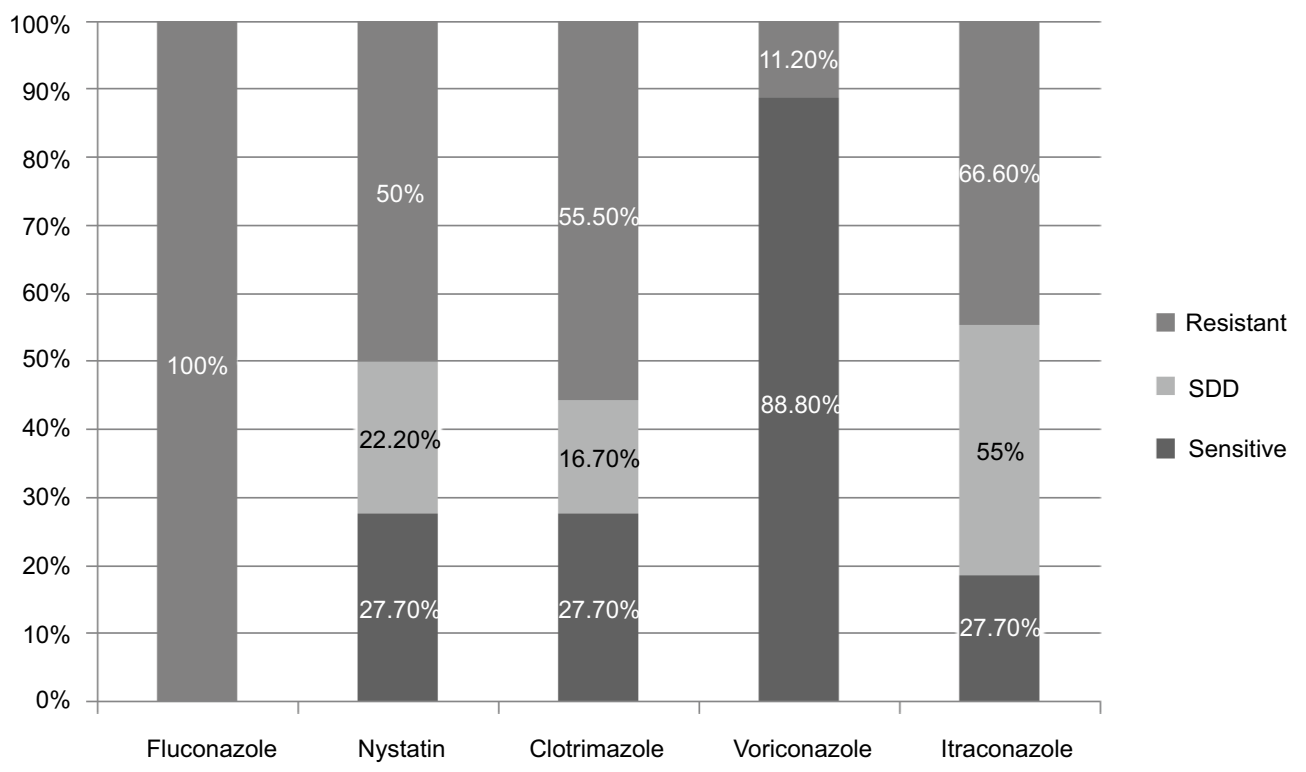

Figure 5 Antifungal susceptibility pattern in Candida krusei.

Abbreviation: SDD, susceptible dose dependent.

(25\%) of the isolated C. glabrata, SDD in five $(31.25 \%)$ of the isolated C. glabrata and resistant in seven (43.7\%) of the isolated C. glabrata (Figure 6).

Susceptibility of 11 isolates of $C$. dubliniensis with respect to each of the antifungal was analyzed as follows: fluconazole was sensitive in two (18.2\%) of the patients, SDD in one $(9.1 \%)$ of the patient and resistant in eight $(72.7 \%)$ of the patients isolated with $C$. dubliniensis. Nystatin was sensitive in none of the isolated $C$. dubliniensis, SDD in none of the isolated C. dubliniensis and resistant in $11(100 \%)$ of the isolated $C$. dubliniensis. Clotrimazole was sensitive in none of the isolated $C$. dubliniensis, SDD in one (9.1\%) of the isolated $C$. dubliniensis and resistant in $10(90.9 \%)$ of the isolated C. dubliniensis. Voriconazole was sensitive in nine (81.8\%) of the isolated C. dubliniensis, SDD in none of the isolated C. dubliniensis and resistant in two (18.2\%) of the isolated $C$. dubliniensis. Itraconazole was sensitive in two (18.2\%) of the isolated C. dubliniensis, SDD in one (9.1\%) of the isolated C. dubliniensis and resistant in eight (72.7\%) of the isolated C. dubliniensis (Figure 7). 


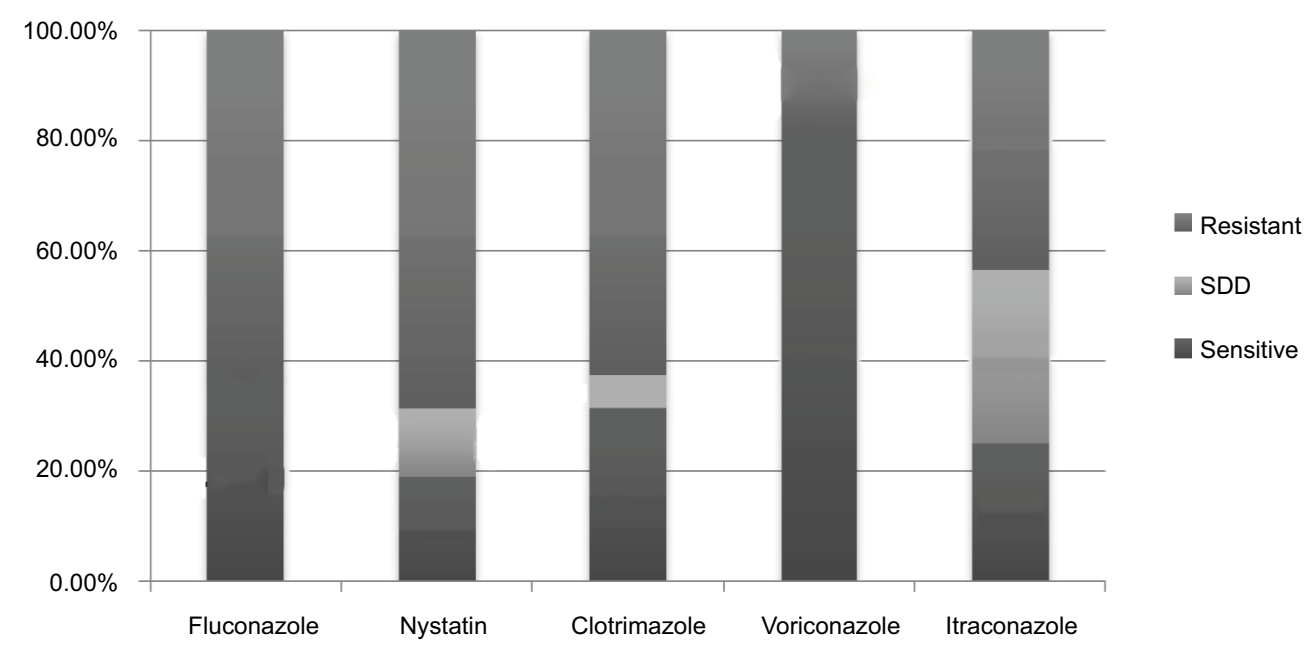

Figure 6 Antifungal susceptibility pattern in Candida glabrata.

Abbreviation: SDD, susceptible dose dependent.

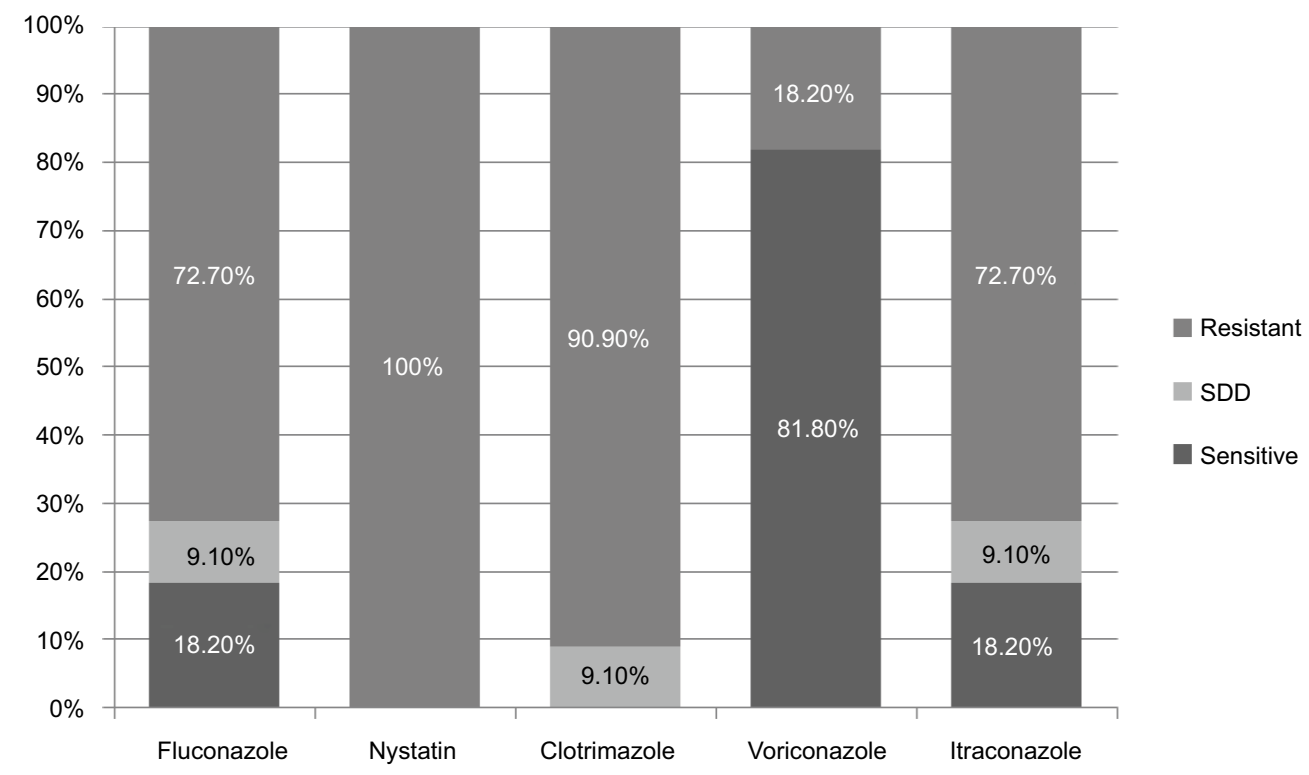

Figure 7 Antifungal susceptibility pattern in Candida dubliniensis.

Abbreviation: SDD, susceptible dose dependent.

\section{Discussion}

Worldwide, many studies have revealed that Candida spp. can switch from a commensal state into a pathogen causing infections, in reaction to transformation in the host leading to infections of oral mucosa, gastrointestinal lining and genital tract epithelium. One of the studies demonstrated that prevalence of candidiasis was more (33.8\%) in women in the age range of 20-29 years, followed by those (24.3\%) in the age range of 30-39 years, and the overall prevalence of VVC was $25 \% .{ }^{13}$ The prevalence rate is parallel to $26 \%$ recorded in Ibadan ${ }^{14}$ and is more or less twofold to that reported in Burkina Faso (14\%) and approximately half to that in (55.4\%) Cameroon. ${ }^{15}$ In another study, the prevalence reported was $30.7 \%$ in Jamaica ${ }^{16}$ and $30 \%$ in Nigeria. ${ }^{17}$ A range of studies have revealed asymptomatic vaginal colonization of Candida as $5-30 \%{ }^{18}$ Many investigators noted a high infection rate in the 20-29 age group, which was consistent with our study, as many of the patients were in the age range of 25-28 years, 
most probably due to indiscriminate drug usage, especially contraceptives. ${ }^{19}$ Various researchers declared women of age group 15-45 years to have the highest incidence, which is almost the same in the present study in women in the age range of 17-44 years. The cause for this high incidence could be due to decreased levels of protective cervical antibodies in the reproductive tract. ${ }^{20}$

Spinillo et $\mathrm{al}^{21}$ reported that a high rate of Candida infections occurs in married women between 30 and 45 years with a reproductive history, repeated sexual activity and taking oral contraceptive pills. Rural women had an elevated rate of infectious vaginitis, owing mostly to conditions of poor medical care, lack of health education, scarce economic resources and difficulty in timely medical treatment. ${ }^{22}$

The presenting complaints in $31 \%$ patients were itching, $29.4 \%$ suffered from vaginal discharge as the primary complaint, $15.6 \%$ of women presented with pain only and $13.3 \%$ presented with a triad of discharge, itching and pain. Among other complaints described were vaginal/vulvar, erythema, dysuria and dyspareunia. In another study, females (55\%) who reported to clinics with VVC had pregnancy. This most likely owes to raised level of hormones during pregnancy, which is an energy source for Candida growth. ${ }^{6}$ An increased propensity to infection is due to both an elevated level of vaginal colonization and a higher prevalence of symptomatic vaginitis. ${ }^{23}$ Our findings were comparable with this study; $19.67 \%$ of $C$. tropicalis was isolated from vaginal swab, which was second to urine. ${ }^{24}$

Oyewole et $\mathrm{a}^{25}$ also detected the highest occurrence of VVC in the second trimester (61\%) of pregnancy, which is almost similar to our study with the highest frequency $(60.2 \%)$ in the second trimester. A Brazilian study reported C. albicans isolated in $92.3 \%$, C. krusei isolated in 3.3\%,C. glabrata isolated in $2.2 \%$, Candida parapsilosis isolated in $1.1 \%$ and $C$. tropicalis isolated in $1.1 \%$ of pregnant women with VVC. ${ }^{26}$ A study in Kenya showed that $C$. albicans was the most frequent isolated (73.7\%) followed by $C$. glabrata (13\%), Candida famata (5\%), C. krusei (3\%) and C. parapsilosis $(1 \%){ }^{27}$

Our findings were somewhat similar to another study publishing the highest occurrence of C. albicans $(54.3 \%)$, followed by C. glabrata $(25.7 \%)$, C. tropicalis $(5.7 \%)$ and C. dublinensis (14.3\%), identified by the CHROMagar test. ${ }^{13}$ Nelson et $\mathrm{l}^{19}$ also reported $C$. albicans as the commonest vaginal species followed by $C$. glabrata in VVC cases in pregnancy. Another report by Oyewole et $\mathrm{al}^{25}$ showed the maximum rate of $C$. albicans as $50 \%$, followed by $C$. glabrata $21.4 \%$, C. tropicalis $14.3 \%$, C. krusei $11.9 \%$ and
Candida pseudotropicalis $2.4 \%$. In colonized women, the predominant species isolated was $C$. albicans, corresponding to $62.9 \%$ from the women with VVC. Other colonizing species in the vaginal mucosa of asymptomatic women were C. glabrata $14.3 \%$, followed by Candida sphaerica $8.6 \%$ and $C$. parapsilosis $2.9 \%{ }^{28}$ In different countries, studies reported C. albicans being most widespread species in VVC (76-89\%), followed by C. glabrata (7-16\%). The proportion of non-albicans spp. related to VVC among these countries varied from $11 \%$ to $24 \% .^{29}$

In India, five Candida species were isolated and identified in a study, where a minor proportion of C. albicans (35.5\%) was noted, followed by increased occurrence of non-albicans species, with C. tropicalis (26.4\%), C. glabrata (20.6\%), C. krusei $(15.7 \%)$ and $C$. dubliniensis $(1.6 \%) .{ }^{30}$ One study has described a higher level of non-C. albicans species occurrence over time. ${ }^{31}$ As stated by Ferrazza et al ${ }^{32}$, almost 25\% of VVC cases were caused by non-albicans species. Comparing the results of our study with those other studies which demonstrated, $C$. glabrata to be the second most significant species among VVC cases because of its higher frequency and increased rate of clinical resistance to antifungals. ${ }^{33}$ In the present study, $41.7 \%$ of VVC cases were due to C. albicans, followed by C. tropicalis (16.7\%), C. krusei (16.7\%), C. glabrata (14.8\%) and C. dubliniensis (10.2\%).

A study in Uganda showed species frequency as follows: C. albicans $78.9 \%$, C. glabrata 14.3\%, C. krusei 3.3\%, C. tropicalis $1.4 \%$, C. famata $0.96 \%$, Candida lusitaniae $0.4 \%$ and $C$. parapsilosis $0.4 \%$. Resistance among antifungals was noted as nystatin $0.61 \%$ of C. albicans. C. krusei showed fluconazole resistance of $71.43 \%$. C. lusitaniae, $C$. krusei, C. famata and C. glabrata were observed to be $100 \%$ resistant to itraconazole. C. famata and C. glabrata showed $50 \%$ and $36.7 \%$ resistance to clotrimazole correspondingly. C. albicans displayed resistance of $20.6 \%$ to itraconazole and $6.6 \%$ to voriconazole. ${ }^{34}$ In an 8-year study, fluconazole resistance increased from $2.4 \%$ to $55.4 \%$ (2006-2012), but the rate dropped to $8.9 \%$ in 2013 . Considering miconazole and itraconazole, resistance increased from $2.4 \%$ and $7.1 \%$ to $59.8 \%$ and $58.9 \%$ (2006-2013), repectively. ${ }^{35}$ Among 100 Candida isolates, C. albicans (30\%), C. parapsilosis (10\%), C. tropicalis (21\%), C. glabrata (8\%), Candida parakrusei $(8 \%)$ and $C$. krusei $(3 \%)$ were isolated by workers. In vitro antifungal activity points to clotrimazole minimum inhibitory concentration of 16 and $8 \mu \mathrm{g} / \mathrm{mL}$ to be effective against $70 \%$ of Candida spp., fluconazole MIC of 64 and $32 \mu \mathrm{g} /$ $\mathrm{mL}$ to be effective against $36.2 \%$ of isolates and nystatin disk to be $63.5 \%$ effective. ${ }^{36}$ In a study conducted in India, 
Candida isolates showed $97.2 \%$ sensitivity to fluconazole, $80 \%$ sensitivity to clotrimazole, $57 \%$ sensitivity to itraconazole and $37 \%$ sensitivity to miconazole. ${ }^{37}$ In most reports, regarding fluconazole and itraconazole, resistance was noted in $42 \%$ and $48 \%$, respectively, of the Candida isolates. ${ }^{28}$ In the current study, C. krusei showed $100 \%$ resistance to fluconazole, followed by $66.6 \%$ resistance to itraconazole. Prostitutes with vulvovaginitis were evaluated in a study that reported an increased susceptibility of $C$. albicans to fluconazole compared to $C$. glabrata. One resistant strain and four SDD strains of C. glabrata were isolated. ${ }^{38}$ On the other hand, when isolates of Candida were tested in a study by Mishra et al ${ }^{39}$, all C. glabrata, $50 \%$ C. tropicalis and $12 \%$ C. albicans isolates were found to be resistant to fluconazole. Recently, some authors reported that among all C. tropicalis isolates, amphotericin B was $100 \%$ sensitive, whereas $37.7 \%$ were found to be resistant to fluconazole, which is similar to the finding in our study with fluconazole resistance of $38.8 \% .^{24}$ Our study indicated that most Candida spp. tested were resistant to fluconazole ( $62 \%)$, followed by clotrimazole (59.3\%), nystatin (58.3\%) and itraconazole (40.7\%); the lowest was recorded for voriconazole $(10.2 \%)$. C. glabrata in our study showed resistance to nystatin $(68.7 \%)$, followed by fluconazole (62.5\%), itraconazole (43.7\%) and voriconazole (18.7\%). The most resistant C. dubliniensis isolate in the current study was nystatin (100\%), followed by clotrimazole $(90.9 \%)$, itraconazole $(72.7 \%)$ and voriconazole $(18.2 \%)$.

\section{Conclusion}

The present study demonstrated the importance of species identification and susceptibility testing for antifungals in pregnant women attending antenatal units. The predominant cause of vulvovaginal candidiasis in this study was C. albicans. An escalating number of Candida spp. from clinical isolates were resistant to antifungal agents that are routinely used for the treatment of VVC due to the fact that majority of the women had previous history of antifungal use. A significant number of non-albicans Candida were recognized, which demonstrated decreased susceptibility to all drugs, particularly the azoles, which are generally used for the management of vaginal candidiasis. Isolation of non-albicans yeasts may have clinical implication due to their reduced susceptibility to various antifungals. Antifungal susceptibility testing may possibly be used to calculate clinical response, to forecast malfunction in management, and accordingly, local antibiograms can aid in empirical assortment of antifungals, guiding options for long-term therapy, and are meant for alternative regimens in testing of isolates from recurrent infections.

\section{Disclosure}

The authors report no conflicts of interest in this work.

\section{References}

1. Pfaller MA, Diekema DJ, International Fungal Surveillance Participant Group. Twelve years of fluconazole in clinical practice: global trends in species distribution and fluconazole susceptibility of bloodstream isolates of Candida. Clin Microbiol Infect. 2004;10(suppl 1):11-23.

2. de Cássia Orlandi Sardi J, de Souza Pitangui N, Gullo FP, e Maria José Soares Mendes Giannini AMFA. A mini review of Candida species in hospital infection: epidemiology, virulence factor and drugs resistance and prophylaxis. Trop Med Surg. 2013;1:141.

3. Anderson M, Karasz A, Friedland S. Are vaginal symptoms ever normal? A review of the literature. MedGenMed. 2004;6(4):49.

4. Lisiak M, Klyszejko C, Pierzchalo T, Marcinkowski Z. Vaginal candidiasis: frequency of occurrence and risk factors. Ginekol Pol. 2000;71(9):964-970.

5. Barousse MM, Espinosa T, Dunlap K, Fidel PL Jr. Vaginal epithelial cell anti-Candida albicans activity is associated with protection against symptomatic vaginal candidiasis. Infect Immun. 2005;73(11):7765-7767.

6. Ragunathan L, Poongothai G, Sinazer AR, et al. Phenotypic characterization and antifungal susceptibility pattern to fluconazole in Candida species isolated from vulvovaginal candidiasis in a tertiary care hospital. J Clin Diagn Res. 2014;8(5):DC01-DC04.

7. Kanagal DV. Prevalence of vaginal candidiasis in pregnancy among coastal south Indian women. J Womens Health Issues Care. 2014;3:6.

8. Parveen N, Munir AA, Din I, Majeed R. Frequency of vaginal candidiasis in pregnant women attending routine antenatal clinic. $J$ Coll Physicians Surg Pak. 2008;18(3):154-157.

9. Spinillo A, Capuzzo E, Gulminetti R, Marone P, Colonna L, Piazzi G. Prevalence of and risk factors for fungal vaginitis caused by non-albicans species. Am J Obstet Gynecol. 1997;176(1):138-141.

10. Rex JH, Pfaller MA, Walsh TJ, et al. Antifungal susceptibility testing: practical aspects and current challenges. Clin Microbiol Rev. 2001;14(4):643-658.

11. Vandenbossche I, Vaneechoutte M, Vandevenne M, De Baere T, Verschraegen G. Susceptibility testing of fluconazole by the NCCLS broth macrodilution method, E-test, and disk diffusion for application in the routine laboratory. J Clin Microbiol. 2002;40(3):918-921.

12. Filler SG, Yeaman MR, Sheppard DC. Tumor necrosis factor inhibition and invasive fungal infections. Clin Infect Dis. 2005;41(3):208-212.

13. Nurat AA, Babalola GO, Shittu MO, Tijani MA, Adekola SA. Detection and epidemiology of vulvovaginal candidiasis among asymptomatic pregnant women attending a tertiary hospital in Ogbomoso, Nigeria. Int J Biomed Res. 2015;6(7):518-523.

14. Anorlu R, Imosemi D, Odunukwe N, Abudu O, Otuonye M. Prevalence of HIV among women with vaginal discharge in a gynecological clinic. J Natl Med Assoc. 2004;96(3):367.

15. Toua V, Djaouda M, Gaké B, et al. Prevalence of vulvovaginal candidiasis amongst pregnant women in Maroua (Cameroon) and the sensitivity of Candida albicans to extracts of six locally used antifungal plants. Int Res J Microbiol. 2013;4(3):89-97.

16. Kamara P, Hylton-Kong T, Brathwaite A, et al. Vaginal infections in pregnant women in Jamaica: prevalence and risk factors. Int $J$ STD AIDS. 2000;11(8):516-520.

17. Okonkwo N, Umeanaeto P. Prevalence of vaginal candidiasis among pregnant women in Nnewi Town of Anambra State, Nigeria. Afr Res Rev. 2010;4(4):539-548.

18. Beigi RH, Meyn LA, Moore DM, Krohn MA, Hillier SL. Vaginal yeast colonization in nonpregnant women: a longitudinal study. Obstet Gynecol. 2004;104(5, pt 1):926-930.

19. Nelson M, Wanjiru W, Margaret MW. Prevalence of vaginal candidiasis and determination of the occurrence of Candida species in pregnant women attending the antenatal clinic of Thika District Hospital, Kenya. Open J Med Microbiol. 2013;2013:264-272. 
20. Sobel JD, Faro S, Force RW, et al. Vulvovaginal candidiasis: epidemiologic, diagnostic, and therapeutic considerations. Am J Obstet Gynecol. 1998;178(2):203-211.

21. Spinillo A, Capuzzo E, Nicola S, Baltaro F, Ferrari A, Monaco A. The impact of oral contraception on vulvovaginal candidiasis. Contraception. 1995;51(5):293-297.

22. Mulu W, Yimer M, Zenebe Y, Abera B. Common causes of vaginal infections and antibiotic susceptibility of aerobic bacterial isolates in women of reproductive age attending at Felegehiwot referral hospital, Ethiopia: a cross sectional study. BMC Womens Health. 2015;15(1):42.

23. Neerja J, Aruna A, Paramjeet G. Significance of Candida culture in women with vulvovaginal symptoms. J Obstet Gynecol India. 2006;56(2):139-141.

24. Yesudhason BL, Mohanra MK. Candida tropicalis as a predominant isolate from clinical specimens and its antifungal susceptibility pattern in a tertiary care hospital in Southern India. J Clin Diagn Res. 2015;9(7):14.

25. Oyewole O, Okoliegbe I, Alkhalil S, Isah P. Prevalence of vaginal candidiasis among pregnant women attending federal university of technology, Minna, Nigeria, Bosso clinic. Res J Pharm Biol Chem Sci. 2013;4(1):113-120.

26. Dias LB, Melhem MdSC, Szeszs MW, Meirelles Filho J, Hahn RC. Vulvovaginal candidiasis in Mato Grosso, Brazil: pregnancy status, causative species and drugs tests. Braz J Microbiol. 2011;42(4):1300-1307.

27. Mutua F, Revathi G, Machoki J. Species distribution and antifungal sensitivity patterns of vaginal yeasts. East Afr Med J. 2010;87(4):156-162.

28. Brandolt TM, Klafke GB, Gonçalves CV, et al. Prevalence of Candida spp. in cervical-vaginal samples and the in vitro susceptibility of isolates. Braz J Microbiol. 2017;48(1):145-150.

29. Corsello S, Spinillo A, Osnengo G, et al. An epidemiological survey of vulvovaginal candidiasis in Italy. Eur J Obstet Gynecol Reprod Biol. 2003;110(1):66-72.

30. Babin D, Kotigadde S, Rao PS, Rao T. Clinico-mycological profile of vaginal candidiasis in a tertiary care hospital in Kerala. Int J Res Biol Sci. 2013;3(1):55-59.
31. Regulez P, Garcia Fernandez J, Moragues M, Schneider J, Quindos G, Ponton J. Detection of anti-Candida albicans IgE antibodies in vaginal washes from patients with acute vulvovaginal candidiasis. Gynecol Obstet Invest. 1994;37(2):110-114.

32. Ferrazza M, Maluf MLF, Consolaro MEL, Shinobu CS, Svidzinski TIE, Batista MR. Caracterização de leveduras isoladas da vagina e sua associação com candidíase vulvovaginal em duas cidades do sul do Brasil. [Characterization of yeasts isolated from the vagina association with vulvovaginal candidiasis in two cities in southern Brazil]. Rev Bras Ginecol Obstet. 2005;27(2):58-63. Portuguese.

33. Richter SS, Galask RP, Messer SA, Hollis RJ, Diekema DJ, Pfaller MA. Antifungal susceptibilities of Candida species causing vulvovaginitis and epidemiology of recurrent cases. J Clin Microbiol. 2005;43(5):2155-2162.

34. Mukasa KJ, Herbert I, Daniel A, Sserunkuma KL, Joel B, Frederick B. Antifungal susceptibility patterns of vulvovaginal Candida species among women attending antenatal clinic at Mbarara Regional Referral Hospital, South Western Uganda. Br Microbiol Res J. 2015;5(4):322.

35. Wang F-J, Zhang D, Liu Z-H, Wu W-X, Bai H-H, Dong H-Y. Species distribution and in vitro antifungal susceptibility of vulvovaginal Candida isolates in China. Chin Med J. 2016;129(10):1161.

36. Khan F, Baqai R. In vitro antifungal sensitivity of fluconazole, clotrimazole and nystatin against vaginal candidiasis in females of childbearing age. J Ayub Med Coll Abbottabad. 2010;22(4):197-200.

37. Dharmik PG, Gomashe A, Upadhyay V. Susceptibility pattern of various azoles against Candida species causing vulvovaginal candidiasis. J Obstet Gynaecol India. 2013;63(2):135-137.

38. Otero L, Fleites A, Mendez F, Palacio V, Vázquez F. Susceptibility of Candida species isolated from female prostitutes with vulvovaginitis to antifungal agents and boric acid. Eur J Clin Microbiol Infect Dis. 1999;18(1):59-61.

39. Mishra M, Agrawal S, Raut S, Kurhade A, Powar R. Profile of yeasts isolated from urinary tracts of catheterized patients. J Clin Diagn Res. 2014;8(2):44.
Infection and Drug Resistance

\section{Publish your work in this journal}

Infection and Drug Resistance is an international, peer-reviewed openaccess journal that focuses on the optimal treatment of infection (bacterial, fungal and viral) and the development and institution of preventive strategies to minimize the development and spread of resistance. The journal is specifically concerned with the epidemiology of antibiotic

\section{Dovepress}

resistance and the mechanisms of resistance development and diffusion in both hospitals and the community. The manuscript management system is completely online and includes a very quick and fair peerreview system, which is all easy to use. Visit http://www.dovepress.com/ testimonials.php to read real quotes from published authors. 